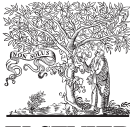

\title{
Gestione anestesiologica e perioperatoria di un caso di sindrome di Brugada
}

Ricevuto il:

14 marzo 2011

Accettato il:

9 maggio 2011

Disponibile online:

$x x x x x x x=$

Anesthesiologic and perioperative management of a case of Brugada syndrome

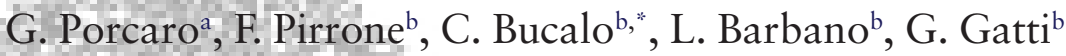

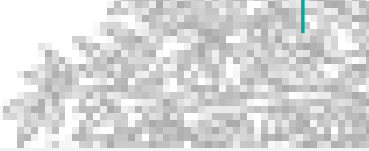

a Specialista in Chirurgia Odontostomatologica, Scuola di Specializzazione in Chirurgia

Odontostomatologica, Università degli Studi Milano-Bicocca Monza (MB)

Specializzando presso la Scuola di Specializzazione in Chirurgia Odontostomatologica,

Università degli Studi Milano-Bicocca Monza (MB)

Parole chiave:

Sindrome di Brugada

Chirurgia orale

Elettrocardiogramma

Gestione perioperatoria

Anestesia locale

Key words:

Brugada syndrome

Oral surgery

Electrocardiogram

Perioperative

management

Local anesthesia

*Autore di riferimento: cettina.bucalo@gmail.com (C. Bucalo)

\section{Riassunto}

Obiettivi: Lo scopo di questo lavoro è descrivere la gestione perioperatoria di un paziente con sindrome di Brugada (SB) sottoposto ad avulsione dentaria in anestesia locale assistita.

Materiali e metodi: Nel seguente caso il cardiologo ha eseguito l'elettrocardiogramma e insieme all'anestesista è stata valutata la corretta gestione anestesiologica e perioperatoria. In seguito, è stato effettuato il trattamento odontoiatrico in sala operatoria.

Risultati: La gestione odontoiatrica del paziente è stata guidata dalla collaborazione di specialisti diversi per fronteggiare eventuali complicanze non gestibili a livello ambulatoriale.

Conclusioni: La SB è una condizione che mette a rischio la vita del paziente e deve essere conosciuta dagli odontoiatri nonostante la sua bassa incidenza perché richiede particolari accorgimenti pre-, intra- e postoperatori.

(c) 2011 Elsevier Srl. Tutti i diritti riservati.

\section{Abstract}

Objectives: To describe the perioperative management of a patient with Brugada syndrome who underwent tooth extraction under assisted local anesthesia.

Materials and methods: In the case reported here, the cardiologist performed an electrocardiogram and selected the form of anesthesia and perioperative management strategy in collaboration with the anesthesiologist. The dental procedure was carried out in the operating room.

Results: This case was managed with a team of different specialists to avoid complications that would not be manageable in a dental practice. Conclusions: Brugada syndrome is a life-threatening condition. Although its incidence is low, dentists must be aware of this syndrome because it requires special pre-, intra-, and postoperative care.

() 2011 Elsevier Srl. All rights reserved. 


\section{G. Porcaro et al.}

\section{CLINICAL IMPLICATIONS}

La sindrome di Brugada è una rara sindrome genetica che può determinare morte improvvisa senza sintomi precedenti o in seguito all'assunzione di sostanze o farmaci, alcuni dei quali utilizzati in odontoiatria. Inoltre, questi pazienti necessitano di una particolare gestione polispecialistica.

Brugada syndrome is a rare genetic syndrome that can cause sudden death, without previous symptoms, or following the use of substances or drugs used in dentistry. These patients need a multispecialist team management.

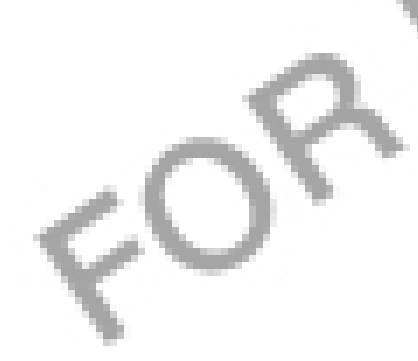

La sindrome di Brugada (SB) è una patologia genetica rara che si presenta con un quadro elettrocardiografico (ECG) tipico e può causare morte improvvisa nei giovani adulti in assenza di alterazioni strutturali del cuore. In letteratura sono tuttavia descritti casi in un ampio range di età (0-77 anni). I sintomi, sincope o morte improwisa, si manifestano più frequentemente nei soggetti di sesso maschile (rapporto maschi-femmine 8:1) [1]. La sindrome è stata descritta per la prima volta da autori italiani nel 1988 sul Giornale Italiano di Cardiologia [2,3], su Mises à Jour Cardiologiques [4] e sull'American Heart Journal [5], ma è conosciuta in tutto il mondo con il nome di "sindrome di Brugada" dal nome dei fratelli Brugada che la descrissero nuovamente nel 1992 [6].

La teoria più diffusa è che si tratti di un'anomalia funzionale su base genetica, a ereditarietà autosomico-dominante a penetranza incompleta, legata a una patologia dei canali del sodio SCN5A il cui gene si trova sul cromosoma 3, canale ionico per la corrente depolarizzante del sodio [7]. Le manifestazioni cliniche, sulla base delle caratteristiche e del grado di malignità delle aritmie alle quali sono correlate, sono diverse. I pazienti affetti da SB, infatti, possono essere del tutto asintomatici, presentare sintomi minori quali cardiopalmo e vertigine o manifestare, nei casi più gravi, sincope e arresto cardiaco [1]. Il quadro ECG può variare dalla semplice extrasistolia ventricolare o sopraventricolare a episodi di tachicardia ventricolare (TV) fino alla fibrillazione ventricolare (FV).

La diagnosi clinica di SB si fonda sull'ECG e sulla presenza di un sopraslivellamento del tratto ST. Poiché quest'ultimo non è presente in condizioni basali e il quadro clinico mostra caratteristiche di transitorietà, è stato individuato un test provocativo impiegando farmaci quali flecainide o ajmalina [8], che permette di slatentizzare il sopraslivellamento nei soggetti affetti.

La SB può determinare morte improvvisa senza sintomi precedenti o in seguito all'assunzione di sostanze o farmaci, alcuni dei quali sono usati in odontoiatria. I farmaci e le condizioni metaboliche da evitare nella SB sono indicati nella tabella / [9]. Come è possibile evincere dalla tabella, i beta-bloccanti, inizialmente testati come farmaci antiaritmici in questi pazienti, devono essere evitati in virtù delle proprietà antiadrenergiche e bradicardizzanti: vari studi hanno infatti ipotizzato che l'aritmia possa instaurarsi con meccanismo bradicardia-dipendente, che giustificherebbe l'alta incidenza di morte cardiaca improvvisa durante la notte [1].

Tra i farmaci sconsigliati usati nella pratica odontoiatrica sono da citare anestetici e vasocostrittori. Dal punto di vista chimico si distinguono due classi principali: gli esteri e le amidi. Gli anestetici locali attualmente impiegati in odontoiatria, come lidocaina, mepivacaina e articaina, appartengono alla classe delle amidi. Anche la bupivacaina, che rientra tra i farmaci da evitare nella SB, è un anestetico locale di tipo amidico, con struttura simile alla mepivacaina, che si caratterizza per un periodo di latenza e per una durata d'azione maggiori, presentando inoltre una maggiore tossicità. Infine, poiché tutti gli anestetici locali causano un certo grado di 


\section{Gestione anestesiologica e perioperatoria di un caso di sindrome di Brugada}

TABELLA I - FARMACI E CONDIZIONI METABOLICHE DA EVITARE NELLA SINDROME DI BRUGADA

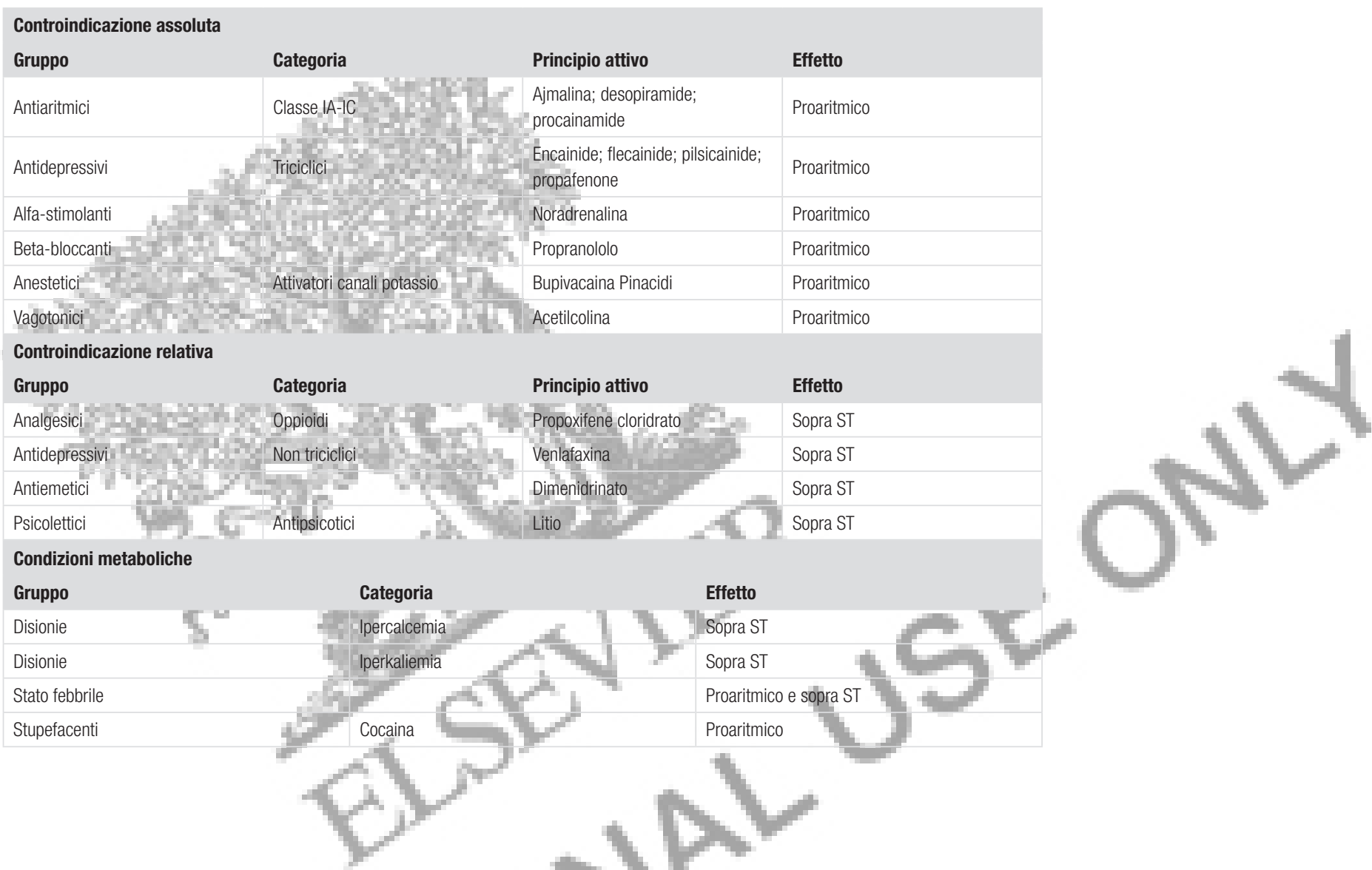

vasodilatazione, l'allontanamento del farmaco dal sito di iniezione può essere diminuito associando un vasocostrittore, come ad esempio l'epinefrina, anch'essa sconsigliata in tale sindrome [10]. Come menzionato, sono stati identificati i farmaci sconsigliati, ma in merito alla terapia, nonostante i numerosi tentativi per identificare un approccio farmacologico efficace, a oggi non è ancora disponibile un trattamento che riduca significativamente il numero e la gravità delle manifestazioni. L'unico presidio in grado di diminuire la mortalità nei pazienti affetti dalla SB è il cardiovertitore-defibrillatore impiantabile (ICD). In presenza di una stratificazione prognostica di rischio intermedio-elevato e qualora manovre "a rischio" assolutamente improcrastinabili, che potrebbero favorire la comparsa di un'aritmia minacciosa, dovessero essere differite, prima andrebbe impiantato l'ICD. È necessario sottolineare inoltre che in presenza di basso rischio le precauzioni riportate non sono necessarie.
Per la stratificazione nei pazienti a rischio medio o basso è stato proposto lo studio elettrofisiologico (SEF) con stimolazione ventricolare programmata per la valutazione dell'inducibilità di $\mathrm{FV}$, ma la riproducibilità del SEF sembra essere bassa.

Di seguito viene presentata una valutazione generale del trattamento anestesiologico e perioperatorio in chirurgia orale di un paziente affetto da SB.

\section{Caso clinico}

Nel maggio 2010, il paziente, di 35 anni, si è presentato presso la Clinica Odontoiatrica dell'Ospedale S. Gerardo di Monza riferendo sintomatologia algica a livello dell'elemento 3.8 che risultava a strette vicinanze del canale alveolare.

In anamnesi, negativa per sincope e sintomi suggestivi di aritmia, è stato segnalato un riscontro occasionale di alterazioni ECG grafiche compatibili 


\section{G. Porcaro et al.}

con SB durante un intervento di tonsillectomia nel settembre 2004 effettuata in anestesia generale. In seguito a tale riscontro, il paziente è stato sottoposto a test da sforzo ed ecocardiogramma, risultati nella norma. Nel marzo 2005, presso il Centro Cardiologico Monzino è stato eseguito il test della flecainide, risultato diagnostico per SB, e il SEF, risultato negativo per aritmia ventricolare.

Il paziente risultava a rischio intermedio, con consigliabile applicazione di ICD di cui però non era portatore.

Fumatore e bevitore sporadico, presentava familiarità negativa per morte improvvisa in età giovanile, ma positiva per patologia ischemica.

Il cardiologo, chiamato a effettuare la visita di parere, ha segnalato alla clinica odontoiatrica l'assenza di controindicazione all'avulsione dentaria e ha indicato la necessità di un monitoraggio ECG durante l'intervento associato all'applicazione delle piastre di un defibrillatore.

Prima del trattamento odontoiatrico, dopo aver ottenuto il consenso informato da parte del paziente, sono stati effettuati gli esami ematochimici e l'ECG (fig. 1). ॥ referto del tracciato elettrocardiografico ha indicato:

- ritmo sinusale a FC di 60 bpm;

- asse QRS normo-orientato a circa 45\%;

- intervallo PR nella norma (148 msec);

- intervallo QTC nella norma (404 msec)

- transizione R/S anticipata in V2;

- normale progressione dell'onda R nelle precordiali;
- sopraslivellamento del tratto ST in V1-V2-V3, con onda J presente in V1 e V2. In V2, il sopraslivellamento del tratto ST assume una morfologia concava (saddle back) di circa $2 \mathrm{~mm}$, da pattern simil-Brugada tipo 2;

- inversione asimmetrica dell'onda T in V1 (nel $20 \%$ circa dei soggetti tale inversione in V1 può essere presente in assenza di patologia);

- ECG nei limiti della norma.

In seguito alla valutazione cardiologica dell'ECG è stata effettuata l'analisi della gestione perioperatoria e anestesiologica. È stata consigliata un'anestesia locale assistita che consentisse di evitare la somministrazione di elevati dosaggi di anestetici (che possono deprimere la funzionalità cardiovascolare e respiratoria). II paziente, infatti, presentava rischio intermedio per eventi aritmici, ma rischio anestesiologico elevato con un valore ASA 3, con complicanze aggiuntive quali l'arresto cardiocircolatorio per FV/ TV. Per tale ragione, è risultata indicata l'applicazione delle piastre del defibrillatore e sconsigliato l'uso di farmaci proaritmici, inclusi bupivacaina e noradrenalina.

In fase preoperatoria sono state somministrate una terapia infusionale di soluzione polisalina 500 per il controllo degli elettroliti e una premedicazione con Valium 20 e profilassi ab ingestis. II monitoraggio ECG è stato predisposto per l'intera durata dell'intervento applicando gli appositi elettrodi per contrastare un'eventuale FV. È stato impiegato un defibrillatore a corrente bifasica.

Fig. 1

ECG del paziente trattato.
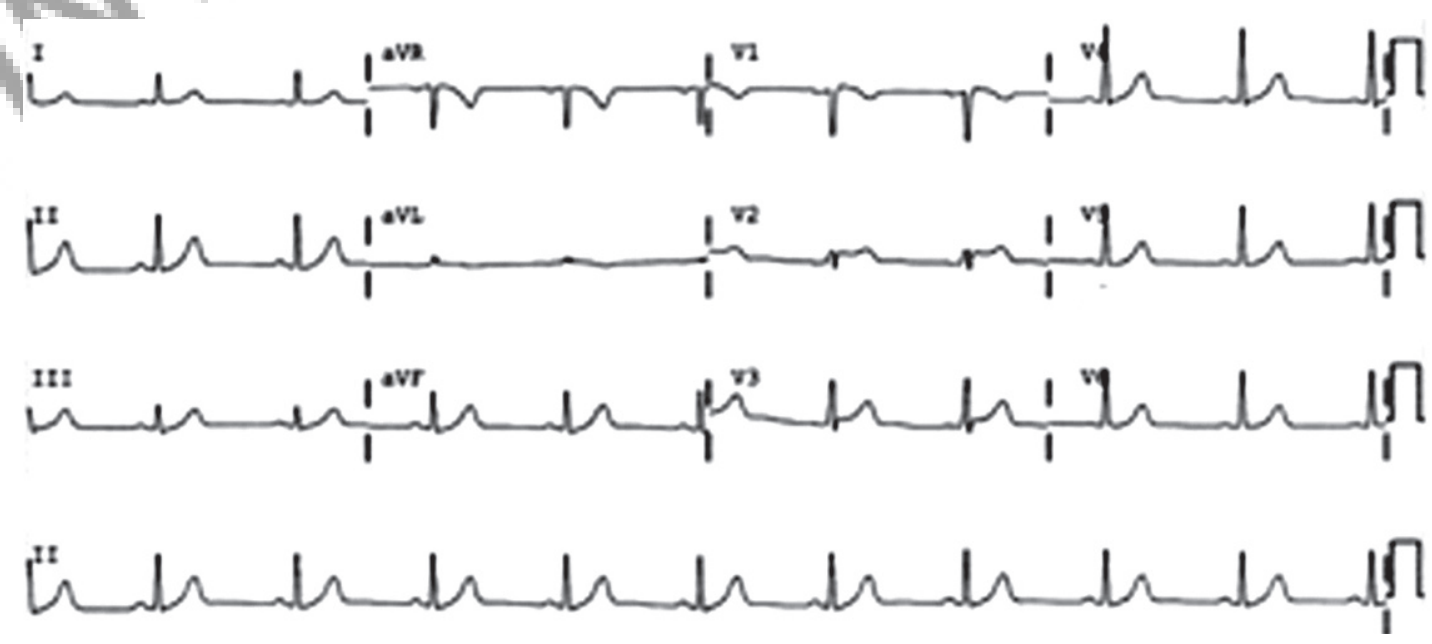


\section{Gestione anestesiologica e perioperatoria di un caso di sindrome di Brugada}

È stata usata lidocaina, un anestetico locale di tipo amidico che produce un'anestesia più rapida, più intensa, e più duratura di procaina, usata sia nelle anestesie locali sia come antiaritmico. Non è allergenica, né cardiotossica, né rientra tra i farmaci da evitare nella SB perché si tratta di un antiaritmico di classe IB, che pare non determinare un sopraslivellamento del tratto ST in questi pazienti [11]. L'intervento di avulsione dentaria è durato circa 1,5 ore e ha previsto la coronotomia, la rizectomia e l'utilizzo del piezosurgery per la vicinanza al canale alveolare (figg. 2-6). II postoperatorio è risultato nella norma (PA 130/80 mmHg e FC 66 bpm). Infine, è stato effettuato il controllo degli elettroliti e somministrata la terapia post-chirurgica con Augmentin, $2 \mathrm{~g}$, e desametasone, $4+4 \mathrm{mg}$. Al bisogno è stata prescritta una terapia analgesica con FANS o paracetamolo. Tra i provvedimenti necessari va sottolineato che l'assistenza postoperatoria è stata condotta in Terapia Intensiva.

Gli autori dichiarano che lo studio presentato è stato realizzato in accordo con gli standard etici stabiliti nella Dichiarazione di Helsinki e che il consenso informato è stato ottenuto da tutti i partecipanti prima del loro arruolamento allo studio.

\section{Discussione}

La maggior parte dei dati pubblicati in merito alla gestione anestesiologica del paziente con SB riguarda l'anestesia generale [11]. La somministrazione di propofol, fentanyl e ossigeno oppure propofol e midazolam oppure propofol, sevoflurano e fentanyl ha mostrato di non slatentizzare la sindrome [12]. Molti articoli indicano la necessità di evitare alcuni farmaci comunemente usati in chirurgia orale, come bupivacaina, che, secondo Veernoy et al. [13], potrebbero indurre manifestazioni aritmiche in pazienti portatori silenti di mutazioni dei canali SCN5A. Altri studi, come quello di Barajas-Martinez et al. [14], hanno dimostrato che la somministrazione di lidocaina può determinare un particolare pattern ECG in rari casi in cui è presente una doppia mutazione dei canali cardiaci del sodio.

Una review dei dati pubblicati ha indicato alcuni principi comuni e farmaci che sono raccomandati per il trattamento perioperatorio di questi pazienti [12]: applicazione di un defibrillatore esterno bifasico; monitoraggio ECG durante l'intervento; monitoraggio continuo della PA; evitare i farmaci sconsigliati; prestare attenzione alla gestione postoperatoria di febbre, nausea e vomito.

Nei pazienti in anestesia generale occorre utilizzare atropina ed efedrina per diminuire il tono vagale e isoproterenolo per migliorare l'elevazione del tratto

Figg. 2 e 3

TC e Ortopantomografia.

Fig. 2

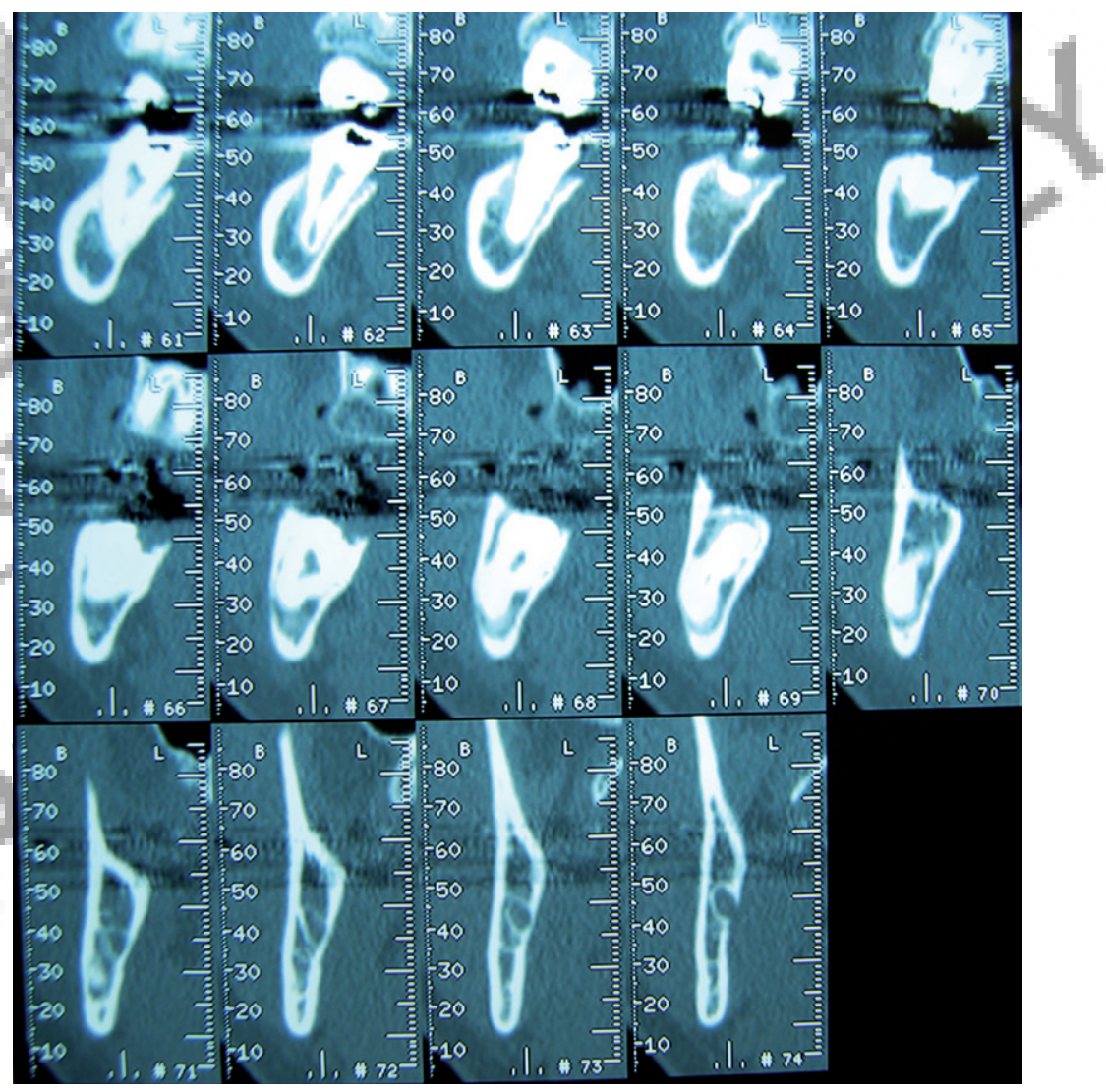

Fig. 3

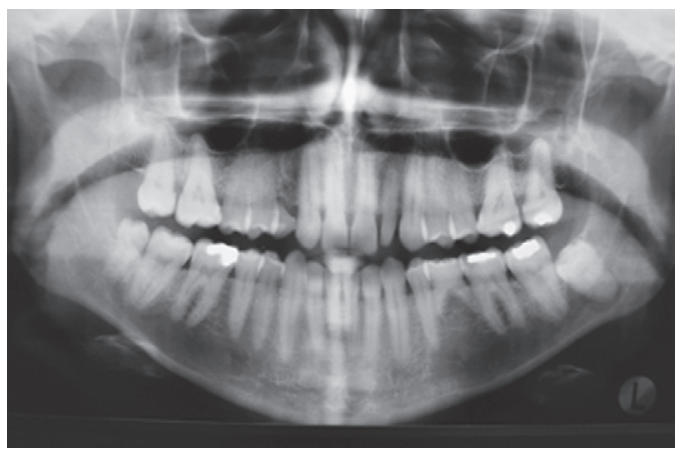




\section{G. Porcaro et al.}

Figg. 4-6

Situazione pre-, intrae postoperatoria.
Fig. 4

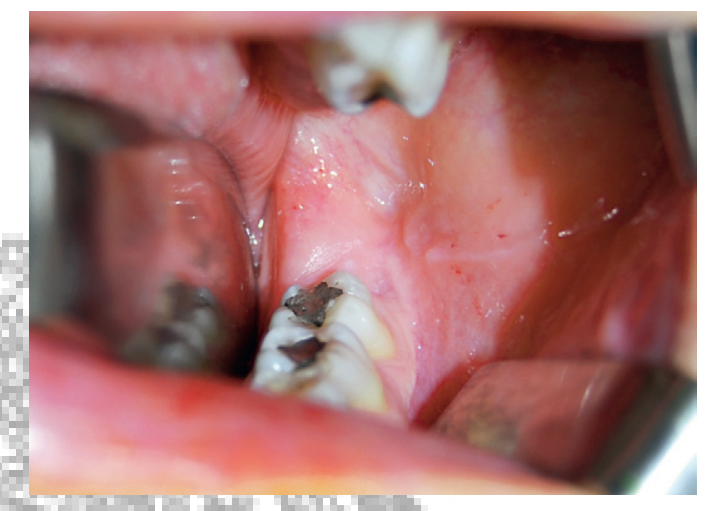

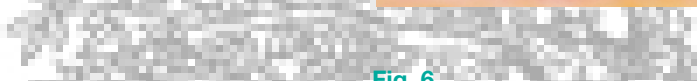

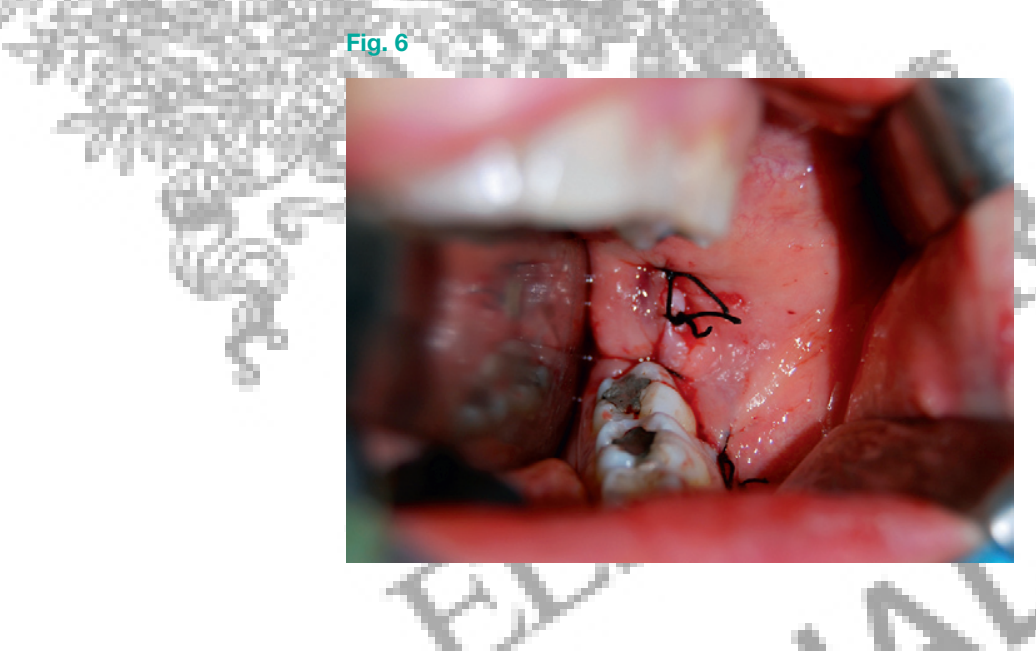

ST. Nei pazienti portatori di ICD, in accordo con il cardiologo, il dispositivo deve essere spento prima della chirurgia e riattivato alla fine dell'intervento [15].

II monitoraggio va effettuato in Terapia Intensiva per 24 ore a causa delle variazioni del pattern ST [16].

\section{Conclusioni}

La SB è una condizione che mette a rischio la vita del paziente e deve essere conosciuta dagli odontoiatri nonostante la sua bassa incidenza perché richiede particolari accorgimenti pre-, intra- e postoperatori. In primo luogo, è assolutamente necessaria la comunicazione fra l'odontoiatra e il cardiologo che si occupa della valutazione del rischio del paziente e deve indicare il trattamento più sicuro ed efficace. Inoltre, il cardiologo e l'anestesista devono fare una scelta accurata per quanto riguarda il tipo di anestesia da somministrare sulla base della valutazione generale del paziente. L'odontoiatra, inoltre,
Fig. 5

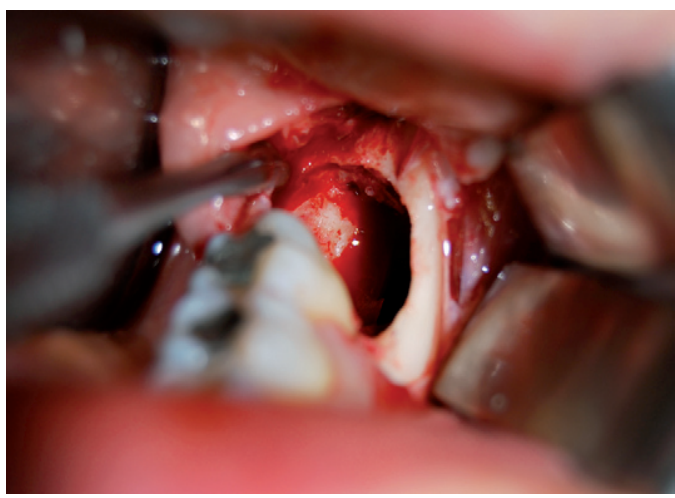

deve prestare estrema attenzione all'anamnesi per valutare se il paziente indica familiarità per morte improvvisa o patologia ischemica, così da poter essere indirizzato a eseguire ulteriori accertamenti prima della terapia odontoiatrica stessa.

Le linee guida e gli accorgimenti sopra descritti permettono all'équipe di sottoporre il paziente a una più corretta e sicura gestione anestesiologica e chirurgica.

\section{Conflitto di interessi}

Gli autori dichiarano di non aver nessun conflitto di interessi.

\section{Finanziamento allo studio}

Gli autori dichiarano di non aver ricevuto finanziamenti istituzionali per il presente studio.

\section{Ringraziamenti}

Si ringraziano tutti i collaboratori che hanno reso possibile l'esecuzione di questo caso clinico.

\section{Bibliografia}

1. Grillo M, Napolitano C, Bloise R, Priori SG. La sindrome di Brugada: epidemiologia, stratificazione del rischio e management clinico. Ital Heart J 2002;3(Suppl):919-27. 


\section{Gestione anestesiologica e perioperatoria di un caso di sindrome di Brugada}

2. Nava A, Canciani B, Martini B, La ripolarizzazione precoce nelle precordiali destre. Correlazioni ECG-

VCGelettrofisiologia. G Ital Cardiol 1988;18(suppl 1):118. 3. Martini B, Nava A, Buja GF, Fibrillazione ventricolare in apparente assenza di cardiopatia. Descrizione di sei casi. G Ital Cardiol 1988;18(suppl 1):136. Abstract. 4. Nava A, Canciani B, Schiavinato ML, Martini B. La repolarisation precoce dans le precordiales droites: trouble de la conduction intraventriculaire droite. Correlations de l'electrocardiographie-vectorcardiographie avec l'electrophysiologie. Mises a Jour Cardiologiques 1988;17:157-9. 5. Martini-B, Nava A, Thiene G, Ventricular fibrillation without apparent heart disease: description of six cases. Am Heart J 1989;118:1203-9.

6. Brugada P, Brugada J. Right bundle-branch block, persistent ST segment elevation and sudden cardiac death: a distinct clinical and electrocardiographic syndrome. J Am Coll Cardiol 1992;20:1391-6.

7. Wang Q, Shen J, Splawsky I, SCN5A mutation associated with inherited cardiac arrhytmia, long QT syndrome. Cell 1995;80:805-11.

8. Brugada J, Brugada R, Brugada P. Right bundle-branch block and ST-segment elevation in leads V1 through V3: a marker for sudden death in patients without demonstrable structural heart disease. Circulation 1998:97:457-60.

9. Eckardt L, Probst V, Smits JP, Bahr ES, Wolpert C,

Schimpf $\mathrm{R}$, et al. Long-term prognosis of individuals with right precordial ST-segment-elevation Brugada syndrome. Circulation 2005;111:257-63.

10. Theodotou N, Cillo Jr JE. Brugada syndrome (sudden unexpected death syndrome): perioperative and anesthetic management in oral and maxillofacial surgery. J Oral Maxillofac Surg 2009;67:2021-5.

11. Hines R, Marschall K. Stoelting's anesthesia and co-existing disease. 5th edition Philadelphia: Churchill Livingstone; 2008.

12. Inamura M, Okamoto H, Kuroiwa M, Hoka S. General anesthesia for patients with Brugada syndrome: a report of six cases. Can J Anaesth 2005;52:409-12.

13. Vernooy K, Sicouri S, Dumaine R, Hong K, Oliva A, Burashnikov E, et al. Genetic and biophysical basis for bupivacaine-induced ST segment elevation and VTNF. Anesthesia unmasked Brugada syndrome. Heart Rhythm 2006;3:1074-8.

14. Barajas-Martínez HM, Hu D, Cordeiro JM, Wu Y,

Kovacs RJ, Meltser H, et al. Lidocaine-induced Brugada syndrome phenotype linked to a novel double mutation in the cardiac sodium channel. Circ Res 2008;103:396-404. 15. Stone KR, McPherson CA. Assessment and management of patients with pacemakers and implantable cardioverter defibrillators. Crit Care Med 2004;32:S155-65.

16. Vaccarella A, Vitale P, Presti CA. General anaesthesia in a patient affected by Brugada syndrome. Minerva Anestesiol 2008; 74:149-52. 